\title{
Cardiovascular Emergencies
}

Tn the ED, cardiovascular diseases are an everyday life-threatening occurrence. However, with proper assessment and fast treatment, cardiac diseases are resolved every day in EDs across the country. After studying this chapter, you will have a basic understanding of cardiovascular assessments and treatments. This chapter does not replace EKG courses or the advanced cardiovascular life support certification required to work in the ED. Many nurses find keeping an advanced cardiovascular life support handbook for study very helpful.

During this part of your orientation, locate and become familiar with:

- EKGs and supplies

- ST-segment elevated myocardial infarction (STEMI) policy and protocol for your facility

- Advanced cardiovascular life support and EKG courses available to you

- Cardiac monitors, defibrillators, and pacers

- Crash carts

- Pacer magnets

- Medications to know: aspirin, alteplase, morphine, nitroglycerin, atropine, adenosine, digoxin, furosemide, calcium channel blockers, beta-blockers, amiodarone, lidocaine, epinephrine, vasopressin, heparin, warfarin, dopamine, nicardipine hydrochloride, and norepinephrine 


\section{CONGESTIVE HEART FAILURE}

In congestive heart failure, the heart fails to pump blood effectively. It can be acute or chronic. As a result, blood backs up. It can back up to the body (right-sided congestive heart failure) or the lungs (leftsided congestive heart failure).

- Causes: Other illnesses can, over time, lead to congestive heart failure. These include hypertension; arrhythmias; diabetes; coronary artery disease; valvular stenosis; cardiomyopathy; emphysema; obesity; pulmonary embolism; anemia; and thyroid disease.

- Signs and symptoms:

- Right sided: Pitting pedal edema; hepatojugular reflux; liver enlargement; nocturia; and jugular vein distention.

- Left sided: Usually develops first; crackles; shortness of breath; pulmonary edema (rales); tachypnea; left ventricular hypertrophy; tachycardia; and ventricular gallop.

- Interventions: Use the mnemonic UNLOAD FAST. Anticipate orders for Upright position, Nitroglycerin, Lasix, Oxygen, Ace inhibitors, Digoxin, Fluid restrictions, Arterial blood gas (ABG), and Sodium restrictions and Tests (digoxin levels, ABG, and metabolic panel). Also establish intravenous (IV) access; monitor cardiac performance; and monitor intake and output. Provide condom catheter, external female catheter, urinal, bedside commode, or bedpan for frequent urination after furosemide administration.

Notes:

Question: Which patient position is best to hear S3 (ventricular gallop) and S4 (atrial gallop)?

Answer: Left lateral. 


\section{ACUTE MYOCARDIAL INFARCTION}

Acute myocardial infarction is the result of a clogged coronary artery supplying blood to the heart muscle. The patient's history often reveals hypertension, coronary artery disease, high cholesterol, and smoking (see Figure 3.1).

- Causes: Blood clots; coronary arterial spasm from cocaine use; contributing factors: hypertension; coronary artery disease; smoking; obesity; hyperlipidemia; and genetics.

- Signs and symptoms: Nausea and vomiting; diaphoresis; shortness of breath; fatigue; anxiety; hypertension or hypotension; and chest pain (described often as pressure, squeezing, tightness, or vague) that may radiate to the left shoulder or jaw. Females may present with vague weakness, fatigue, and dyspnea. EKG may or may not reveal ST segment elevation.

- Interventions: MOVE!! (Monitor, Oxygen, Venous access [2 large bore], and EKG within 7 minutes); anticipate orders to administer medications MONA (Morphine, Oxygen, Nitroglycerin sublingual (SL), and Aspirin); obtain cardiac enzymes; arrange for chest $\mathrm{x}$-ray and cardiologist consult; prepare for possible cardiac cath lab admission or thrombolytics (alteplase), anticoagulant (heparin) therapy; and reassess/monitor chest pain.

Notes:

Question: What factors absolutely contraindicate the use of thrombolytics?

Answer: Active bleeding, recent surgery, or recent trauma.

Question: What test should be done prior to administering heparin or thrombolytics?

Answer: Stool hemoccult and coagulant studies.

Question: What is the antidote for heparin?

Answer: Protamine sulfate.

Question: A patient allergic to fish might also be allergic to what medication?

Answer: Protamine sulfate. It is derived from salmon sperm. 


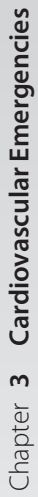

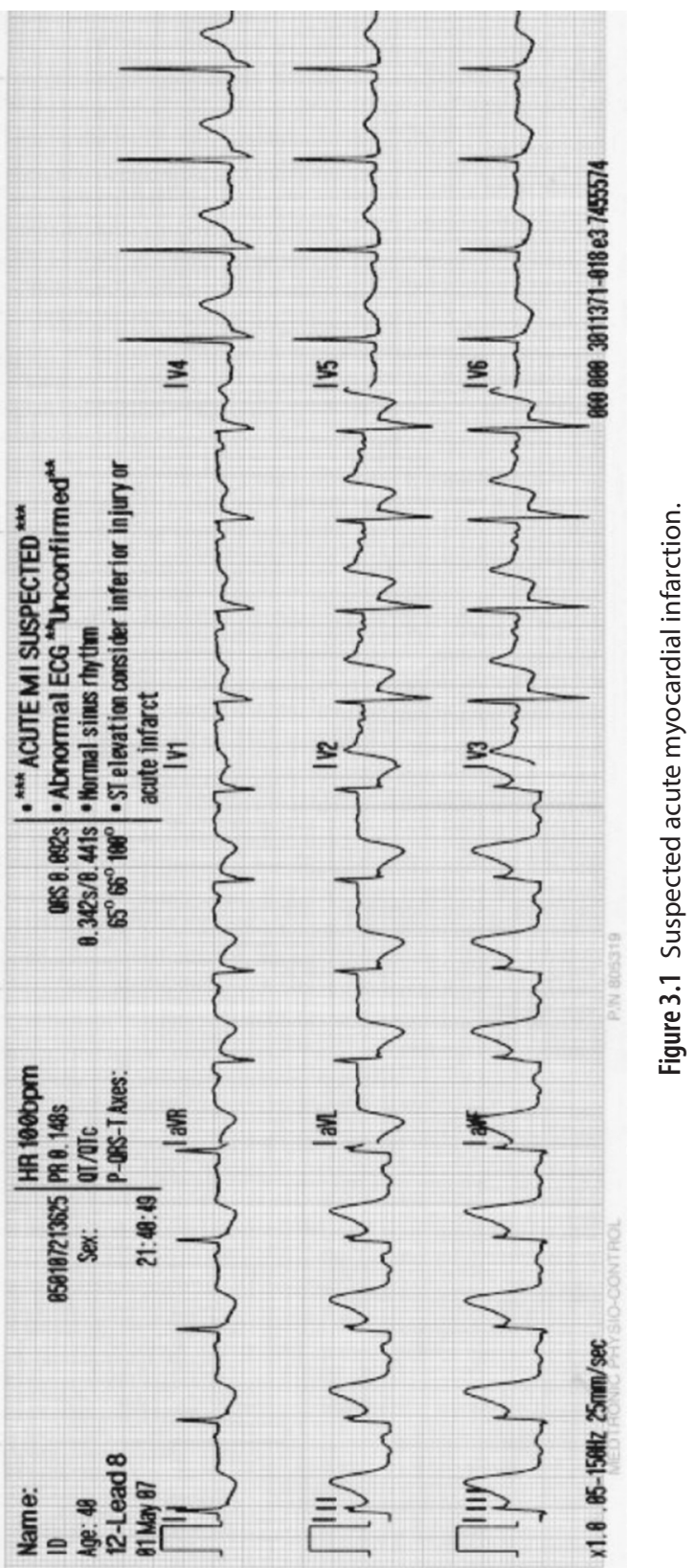




\section{ARTERIAL OCCLUSION}

Arterial occlusion means a clogged artery.

- Causes: Coronary artery disease; atherosclerosis; hypertension; smoking; and hyperlipidemia.

- Signs and symptoms: $\mathrm{Cool} / \mathrm{pale}$ extremities and weak pulse to the affected extremity.

- Interventions: Maintain extremity in dependent position; assess pulses through Doppler ultrasound; and prepare for possible surgery.

Notes: 


\section{ENDOCARDITIS}

Endocarditis is an infection of the inner lining of the heart and/or the heart valves.

- Causes: Endocarditis occurs when a person with faulty heart valves contracts a common bacterial infection. For example, a bacterial infection in the skin can travel through the blood and attach to the faulty heart valve, resulting in endocarditis.

- Signs and symptoms: Petechiae, Shortness of breath, Fever and chills, Roth's spots, Osler's nodes, systolic Murmur, Janeway lesions (red spots on soles of feet), Anemia, Nail bed splinter hemorrhaging; Emboli, and chest pain. To remember these symptoms, just remember "PS: FROM JANE."

- Interventions: Anticipate orders to obtain multiple blood cultures from multiple sites, administer antibiotics intravenously, and do a complete blood count.

Notes: 


\section{AORTIC INJURIES}

Aortic injuries may occur anywhere on the ascending aorta, aortic arch, descending thoracic aorta, or abdominal aorta. The injuries can result in aneurysm, tear, or rupture. Without immediate surgery, the patient can bleed to death rapidly. So it is critical for the nurse to identify an aortic injury early.

- Causes: A history of aortic injuries may reveal hypertension; coronary artery disease; congestive heart failure; or a recent chest/abdominal trauma.

- Signs and symptoms (may vary depending on location): Hypotension; loss of consciousness; hypertension in upper extremities; stronger pulse in arms than in legs; tearing chest pain that radiates to the back; tearing abdominal pain; chest wall ecchymosis; and paraplegia.

- Interventions: Get patient on a stretcher; obtain vital signs; check blood pressure in all extremities; notify provider of patient signs and symptoms immediately; prepare for immediate surgery; start two large-bore IV lines; monitor cardiac performance; provide oxygen; perform EKG; and measure pulse oximetry.

Notes:

Question: Which type of trauma most commonly causes a descending thoracic aortic laceration?

Answer: Deceleration trauma that causes shearing. 


\section{SYMPTOMATIC BRADYCARDIA}

In symptomatic bradycardia, heart rate is $<60$ beats per minute resulting in inadequate blood circulation. The patient is symptomatic, displaying signs of poor cardiac perfusion (see Figure 3.2).

- Causes: The cause is not always known, but underlying conditions such as coronary artery disease, heart disease, second- or third-degree heart blocks, hypertension, thyroid disease, medication overdose, and lung disease can contribute to bradycardia.

- Signs and symptoms: Heart rate $<60$ beats per minute; patient looks and feels unwell (e.g., altered loss of consciousness, chest pain, diaphoretic, and pale).

- Interventions: Assess airway, breathing, and circulation (ABCs); provide oxygen; check vital signs; measure pulse oxygen; perform EKG; monitor cardiac performance; anticipate orders to intravenously push $1.0 \mathrm{mg}$ of atropine at 3 - to 5 -minute intervals, establish transcutaneous pacing, administer medications (dopamine or epinephrine), and prepare for transcutaneous (external) or internal pacer.

Notes:

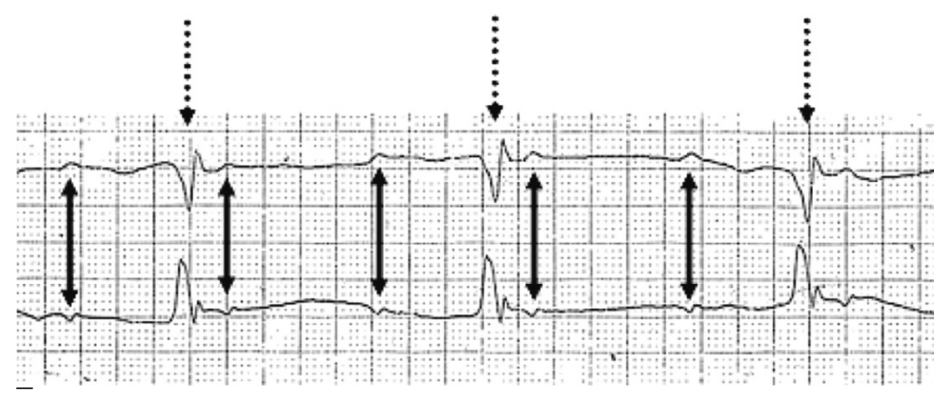

Figure 3.2 Symptomatic bradycardia. 


\section{SUPRAVENTRICULAR TACHYCARDIA}

In supraventricular tachycardia, the heart rate is regular with narrow complexes, but it beats at $>150$ beats per minute. Supraventricular tachycardia can be divided into symptomatic/unstable (patient looks unwell) or asymptomatic/stable (patient looks fine; see Figure 3.3).

- Causes: The cause is not always known. However, some habits and conditions can contribute to it, such as stress, caffeine, smoking, cocaine use, alcohol use, thyroid disease, heart failure, pulmonary embolism, chronic obstructive pulmonary disease, and pneumonia. Some medications for asthma, cold medications, and digoxin can also contribute to supraventricular tachycardia.

- Signs and symptoms: Palpitations; chest pain; diaphoresis; anxiety; and pulse rate $>150$ beats per minute.

- Interventions:

- If patient is symptomatic and unstable, anticipate order to prepare for immediate synchronized cardioversion 50 to $100 \mathrm{~J}$ biphasic.

- If patient is asymptomatic and stable, anticipate orders to attempt vasovagal maneuvers, monitor cardiac performance, open large-bore IV line, provide oxygen, check vital signs, measure pulse oxygen, perform EKG, administer adenosine rapidly by IV push, and slow down atrioventricular (AV) conduction with beta-blockers/calcium channel blockers/ digoxin or amiodarone.

- Give patient a coffee straw and ask the patient to blow through it to assist vagal maneuvers.

Notes: 


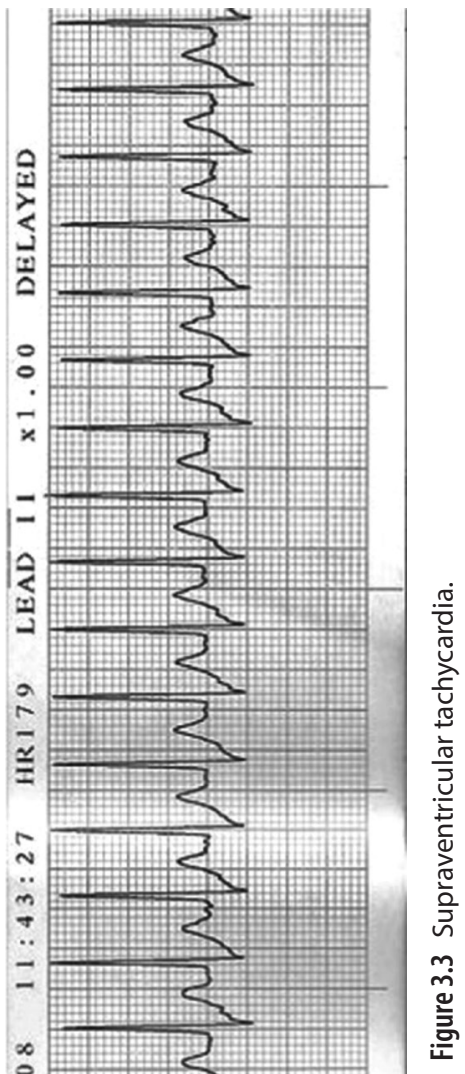

0

$\sum$

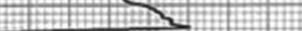
$\longrightarrow+2+$ -

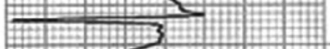

ง

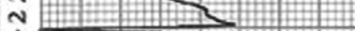

$\Delta$

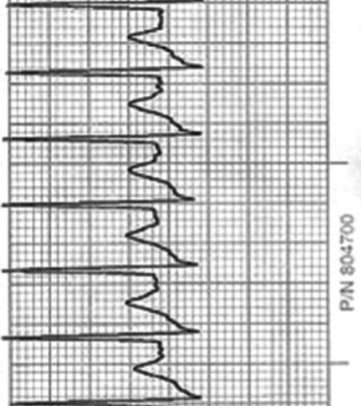

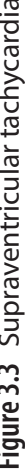




\section{VENTRICULAR FIBRILLATION OR PULSELESS VENTRICULAR TACHYCARDIA}

Ventricular fibrillation (VF) and pulseless ventricular tachycardia (VT) are both irregular rapid rhythms in which there is no pulse (see Figure 3.4 and Table 3.1).

- Causes: Poor cardiac perfusion due to coronary artery disease; shock; hypokalemia; myocardial infarct; or electrocution.

- Signs and symptoms: Decreased level of consciousness; no pulse; VF or VT on cardiac monitor.

- Interventions: Assess for a pulse within 10 seconds; if no pulse present, call for help while initiating CPR starting with chest compressions; do not interrupt chest compressions while applying cardiac monitor/defibrillation pads. Pulseless VT or VF are shockable rhythms. Be sure you know how to set your machine to defibrillation mode and always make sure your team is all clear before pressing the shock button. Anticipate orders to administer early defibrillation, early epinephrine, and amiodarone or lidocaine per advanced cardiovascular life support protocols. Just remember DEAL: Defibrillation, Epinephrine, and Amiodarone or Lidocaine.

Notes:

Table 3.1

Pulseless Ventricular Tachycardia or Ventricular Fibrillation Treatment

1. Shock at $120-200 \mathrm{~J}$ biphasic

- CPR 30/2 for five cycles or $2 \mathrm{~min}$

- Establish IV or IO access and administer epinephrine $1 \mathrm{mg}$ IV/IO during CPR every 3-5 minutes

2. Shock at $200 \mathrm{~J}$ biphasic if still pulseless VT or VF after $2 \mathrm{~min}$ CPR

- CPR 30/2 for five cycles or $2 \mathrm{~min}$

- Amiodarone $300 \mathrm{mg}, 150 \mathrm{mg}$ second dose, or lidocaine during CPR

3. Shock at $200 \mathrm{~J}$ biphasic if still pulseless VT or VF after 2 min CPR

- CPR 30/2 for five cycles or $2 \mathrm{~min}$

- Consider magnesium 1-2 g IV/IO for torsades de pointes

IO, intraosseous; IV, intravenous; VF, ventricular fibrillation; VT, ventricular tachycardia. 


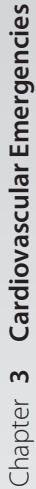
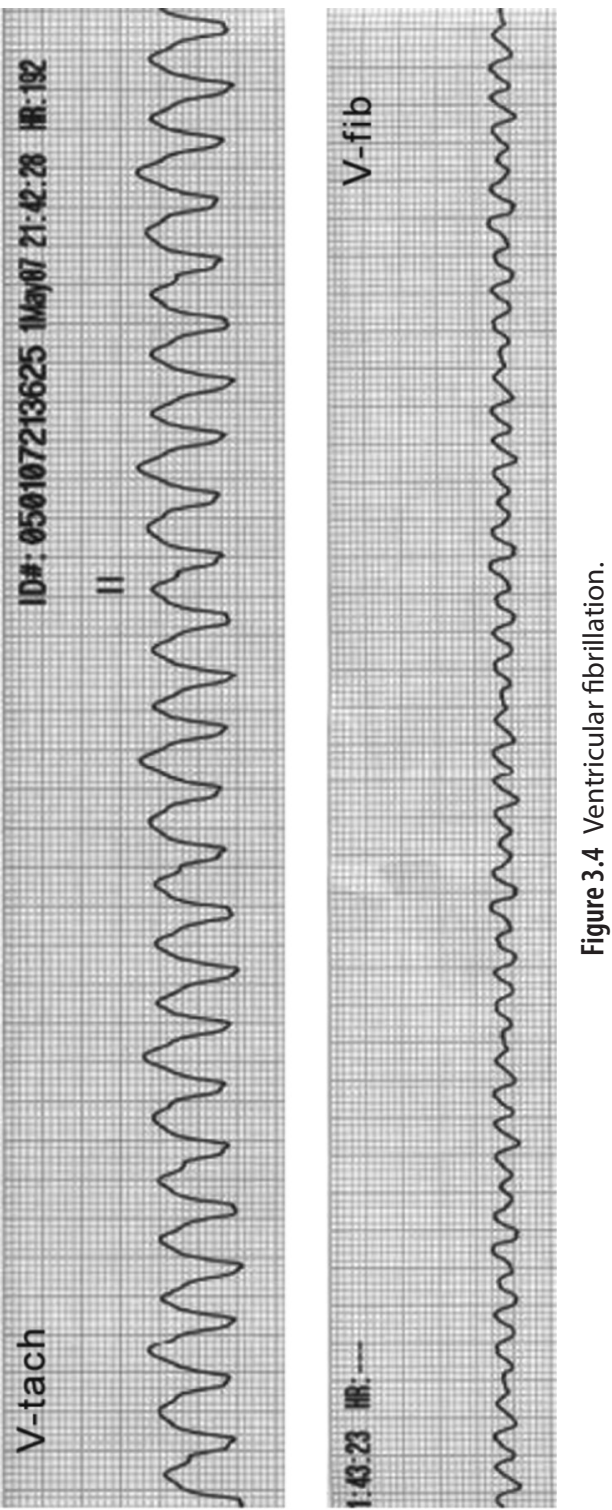
Question: If none of the previously mentioned interventions work, what are some other causes of VF?

Answer: Hypothermia, hypoxia, hypoglycemia, overdose, cardiac tamponade, tension pneumothorax, trauma, acidosis, hypovolemia, and electrolyte imbalances.

Question: You see VF on the monitor, but your patient is asymptomatic, sitting up, and talking to you. What is your first intervention?

Answer: Check for a pulse. You cannot believe everything you see on a monitor; it could be artifact.

Question: What is the maximum number of times one can safely defibrillate a patient?

Answer: There is no limit.

Question: Why do we defibrillate?

Answer: To temporarily produce asystole. This may sound incorrect, but defibrillation actually depolarizes the heart, allowing the natural pacemakers of the heart to kick in.

Question: What dose of lidocaine should be administered to patients with renal failure or liver failure, or patients who are older adults?

Answer: Give half doses of lidocaine to patients with renal failure, patients with liver failure, or patients who are older adults. 


\section{PULSELESS ELECTRICAL ACTIVITY}

Pulseless electrical activity occurs when a rhythm shows on the monitor, but the patient does not have a pulse. Again, you cannot always believe what you see on the monitor.

- Causes: Can be attributed to the five Hs or five Ts.

- Hypovolemia, Hypoxia, Hydrogen ion (acidosis), Hyper-/ hypokalemia, or Hypothermia.

- Toxin (drug overdose), Tamponade/cardiac, Tension pneumothorax, Thrombosis, and Trauma.

- Signs and symptoms: Your patient has no pulse, but there is a rhythm on the monitor. Remember that just because there is electrical activity in the heart does not mean the heart is actually pumping.

- Interventions: Check for a pulse; if no pulse, perform CPR starting with chest compressions; per order, insert an IV line, monitor oxygen, and administer epinephrine. Consider naloxone (Narcan) for opioid overdose and treat the underlying causes.

Notes: 


\section{POST CARDIAC ARREST}

You got them back from the grips of death, so now what do you do? The battle is over, but the war has just begun. Your unstable patient is not really out of the woods so to speak; achieving stability will require a great team effort.

- Causes: Return of spontaneous circulation after cardiopulmonary arrest.

- Signs and symptoms: The patient will have regained a pulse after CPR. In most cases, the patient will remain unresponsive, hypotensive, and in need of respiratory and/or circulatory support.

- Interventions: Document a full primary assessment (ABCs + neurologic). Treat any known underlying causes of cardiopulmonary arrest. Assist ED provider with establishment definitive airway (intubation and ventilator) as needed. Maintain pulse oxygenation between $92 \%$ and $98 \%$ and monitor capnography. Document Glasgow Coma Scale (GCS), pupillary, and complete neurologic assessment. Anticipate provider insertion of central IV line with central venous pressure (CVP) monitoring if available. Anticipate diagnostic test such as ABG, complete blood count (CBC), complete metabolic panel (CMP), Mg, phosphorus, calcium, prothrombin time (PT)/international normalized ratio (INR), partial thromboplastin time (PTT), lactate, creatine kinase $\mathrm{MB}$ (CK-MB), troponin, amylase, lipase, beta human chorionic gonadotropin (hCG) on childbearing women without prior hysterectomy, blood glucose, 12-lead EKG, portable chest X-ray (CXR), and head CT scan. To preserve organs and prevent fever, consider targeted temperature management (TTM). Evaluate if patient meets TTM criteria per hospital protocol such as

- GCS score below 9 to 6 per hospital policy,

- Remaining unresponsive continually for 15 to 30 minutes post cardiac arrest,

- Receiving CPR for $<45$ minutes, and

- NO signs of bleeding or coagulopathies as hypothermia increases bleeding.

If patient meets TTM criteria, obtain head CT prior to attaching the cooling unit to the patient to check for cerebral hemorrhage. Insert core temperature monitoring device (esophageal and/or Foley temperature-sensing probes are more effective than rectal). Attach the cooling unit to the patient according to the manufacture guidelines. Goal-targeted core temperature will be $33{ }^{\circ} \mathrm{C}$ to $36^{\circ} \mathrm{C}$ per order. Typically, patients with cerebral edema will require lower $33^{\circ} \mathrm{C}$, 
otherwise $36{ }^{\circ} \mathrm{C}$ can be just as effective. Goal time for initiation of TTM is within $\mathbf{4}$ hours per policy. Anticipate orders to administer sedatives and paralytics to prevent shivering. Monitor for anticipated bradycardia, hypotension, hypokalemia, and hypoglycemia during TTM. Prepare for ICU admission where rewarming phase will occur very gradually over about 24 to 36 hours.

Question: What is the effect of nitroprusside (Nipride) administered intravenously?

Answer: It reduces afterload and increases cardiac output. It decreases myocardial oxygen demand without affecting the heart rate.

Question: What type of EKG changes might you see in a patient with a potassium level of 7.8 ?

Answer: Bradycardia, peaked T waves, and widened QRS complex.

Question: What equation defines cardiac output?

Answer: Heart rate $\times$ stroke volume.

Question: What are the manifestations of digoxin toxicity?

Answer: Blurred vision, halos, and arrhythmias.

Question: What is the treatment for digoxin toxicity?

Answer: Glucagon, phenytoin (Dilantin), and digoxin immune fab (Digibind).

Question: Name three vasopressors.

Answer: Norepinephrine, dopamine, and metaraminol are vasopressors.

Question: What is the antidote for warfarin (Coumadin)?

Answer: Vitamin $K$.

\section{Fast Facts}

- Heparin affects partial thromboplastin time.

- Warfarin (Coumadin) affects prothrombin time.

- Pro tip: Amiodarone can precipitate when diluted. When hanging an amiodarone drip, be sure to attach an in-line filter to prevent phlebitis. Also check your rate as the dose tapers down after the first 6 hours. 


\section{SUMMARY}

Although cardiovascular diseases threaten lives every day, they can often be resolved with quick treatment. Make EKG a priority for all patients with chest pain. Arrival to EKG should be $<7$ minutes per hospital protocol. Study and understand the various dysrhythmias and their treatments. Print out a rhythm strip on your monitored patients and add it to the patient's chart per shift and as needed. An ED nurse needs to understand the basics of cardiovascular assessments and treatments and be certified in advanced cardiovascular life support. 